\title{
The Repulsive Guidance Molecule RGMa Is Involved in the Formation of Afferent Connections in the Dentate Gyrus
}

\author{
Henriette Brinks, ${ }^{1,2 *}$ Sabine Conrad, ${ }^{4 *}$ Johannes Vogt, ${ }^{2 *}$ Judit Oldekamp, ${ }^{3 *}$ Ana Sierra, ${ }^{5 *}$ Lutz Deitinghoff, ${ }^{7}$ \\ Ingo Bechmann, ${ }^{2}$ Gonzalo Alvarez-Bolado, ${ }^{3 \dagger}$ Bernd Heimrich, ${ }^{2,8}{ }^{\dagger}$ Philippe P. Monnier, ${ }^{6 \dagger}$ Bernhard K. Mueller, ${ }^{7 \dagger}$ and \\ Thomas Skutella ${ }^{1,4}$ \\ ${ }^{1}$ Neuroscience Research Center and ${ }^{2}$ Center for Anatomy, Institute of Cell Biology and Neurobiology, Charité Central Campus, 10098 Berlin, Germany, \\ ${ }^{3}$ Max-Planck-Institute of Experimental Endocrinology, 30625 Hannover, Germany, ${ }^{4}$ Institute of Anatomy, Department of Experimental Embryology, Tissue \\ Engineering Division, and ${ }^{5}$ Department of Ophthalmology, University of Tübingen, 72074 Tübingen, Germany, ${ }^{6}$ Toronto Western Research Institute, \\ Toronto, Ontario, N5T 258 Canada, ${ }^{7} \mathrm{CNS}$ Research, Abbott GmbH and Company KG, 67061 Ludwigshafen, Germany, and ${ }^{8}$ Institute of Anatomy and Cell \\ Biology, University of Freiburg, 79104 Freiburg, Germany
}

In the developing dentate gyrus, afferent fiber projections terminate in distinct laminas. This relies on an accurately regulated spatiotemporal network of guidance molecules. Here, we have analyzed the functional role of the glycosylphosphatidylinositol (GPI)-anchored repulsive guidance molecule RGMa. In situ hybridization in embryonic and postnatal brain showed expression of RGMa in the cornu ammonis and hilus of the hippocampus. In the dentate gyrus, RGM immunostaining was confined to the inner molecular layer, whereas the outer molecular layers targeted by entorhinal fibers remained free. To test the repulsive capacity of RGMa, different setups were used: the stripe and explant outgrowth assays with recombinant RGMa, and entorhino-hippocampal cocultures incubated either with a neutralizing RGMa antibody $(\mathrm{Ab})$ or with the GPI anchor-digesting drug phosphatidylinositol-specific phospholipase C. Entorhinal axons were clearly repelled by RGMa in the stripe and outgrowth assays. After disrupting the RGMa function, the specific laminar termination pattern in entorhino- hippocampal cocultures was lost, and entorhinal axons entered inappropriate hippocampal areas. Our data indicate an important role of RGMa for the layer-specific termination of the perforant pathway as a repulsive signal that compels entorhinal fibers to stay in their correct target zone.

Key words: axon; dentate; development; guidance; hippocampus; neuron

\section{Introduction}

In the hippocampus, extrinsic and intrinsic afferent projections terminate in a nonoverlapping way in distinct laminas. Entorhinal axons terminate on distal dendritic segments of their target neurons in the outer molecular layer of the fascia dentata and stratum lacunosum moleculare of the hippocampus proper. Complementary to the termination patterns of entorhinal axons, hippocampal commissural-associational fibers are restricted to the inner third of the molecular layer of the dentate gyrus and stratum radiatum of the hippocampus proper. This well described anatomical construction implies that specific guidance molecules expressed in the various laminas determine the ingrowth and targeting of these fiber systems. Recent studies on guidance molecules in functional in vitro assays or loss-of-

Received Dec. 1, 2003; revised Feb. 17, 2004; accepted Feb. 18, 2004.

This work was supported by the Deutsche Forschungsgemeinschaft (He 1520/2-1, SK 49/3-3). We thank Jacqueline Mahlo, Lena Schulz, and Annett Wegner for excellent technical assistance. We also thank Kimberly Rosegger and Imke Werner for proof reading.

${ }^{*}$ H.B., S.C., J.V., J.O., and A.S. contributed equally to this work.

${ }^{\dagger}$ G.A.-B., B.H., P.M., and B.K.M. were co-senior authors.

Correspondence should be addressed to Thomas Skutella, Institute of Anatomy, Department of Experimental Embryology, Tissue Engineering Division, Österbergstrasse 3, 72074 Tübingen, Germany. E-mail: tskutella@anatom.uni-tuebingen.de.

D01:10.1523/JNEUROSCI.5296-03.2004

Copyright $\odot 2004$ Society for Neuroscience $\quad 0270-6474 / 04 / 243862-08 \$ 15.00 / 0$ function models have provided direct evidence for an impact of several guidance cues on hippocampal pathfinding and target recognition (Skutella and Nitsch, 2001). A number of diffusible and membrane-associated attractive or repulsive axon guidance molecules have been identified and tested for their role in hippocampal development. These include semaphorins (Chen et al., 2000; Cheng et al., 2001; Pozas et al., 2002; Gu et al., 2003), netrins (Steup et al., 2000; Heimrich et al., 2002), ephrins (Stein et al., 1999), slits (Nguyen Ba-Charvet et al., 1999), and ECM molecules (Förster et al., 2001; Zhao et al., 2003).

The repulsive guidance molecule (RGM), a protein of $33 \mathrm{kDa}$, has been cloned and functionally characterized by Monnier et al. (2002) as a molecular determinant for the retinotectal map formation of the chick embryo (Bayer and Altman, 1987; Walter et al., 1987; Stahl et al., 1990). Three genes in the mouse genome with homology to chick have been isolated (Schmidtmer and Engelkamp, 2004), and their expression patterns have been analyzed (Oldekamp et al., 2004). Mouse RGMa is closely related to chick RGM ( $80 \%$ identity), whereas the two other members of the RGM family are more distantly related. In general, RGMa is a membrane-associated glycoprotein that shares no sequence homology with any other known guidance cues. It is attached to membranes by a glycosylphosphatidylinositol (GPI) anchor. In the chick embryo RGM, mRNA is distributed in an increasing 


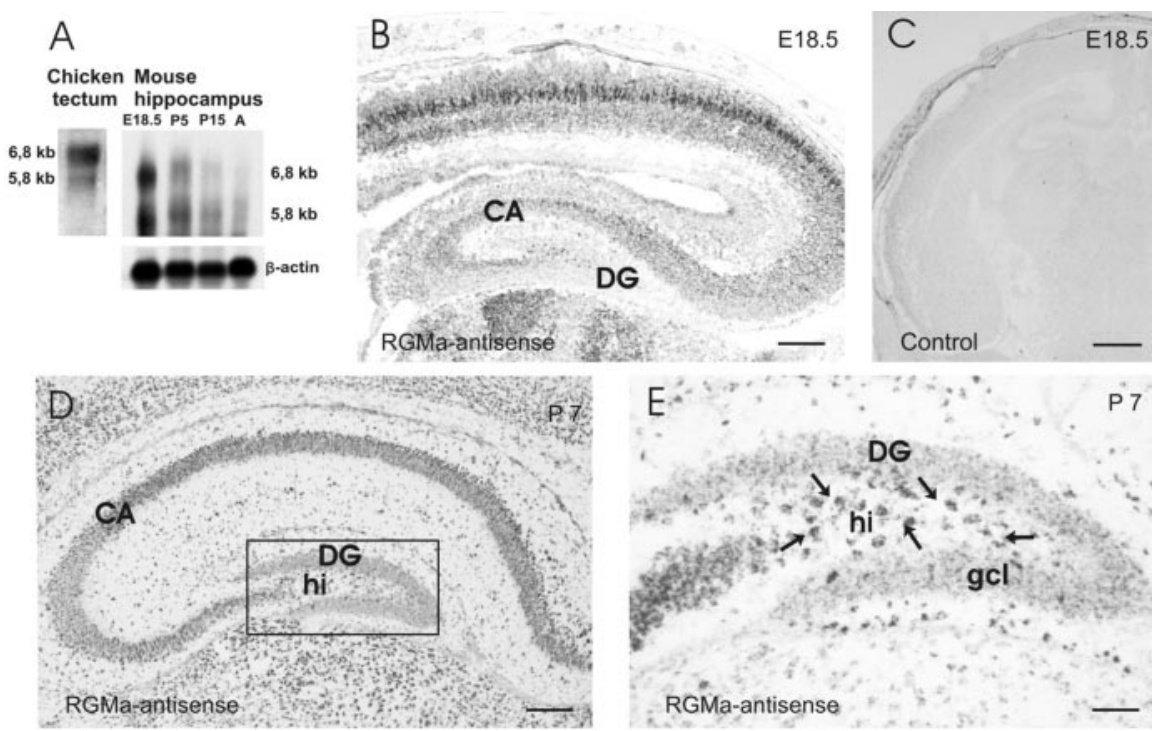

Figure 1. Northern blot analysis of RGMa expression in chicken tectum and mouse hippocampal tissues and distribution of RGMa mRNA in the developing mouse entorhino-hippocampal system. $A$, Chicken embryonic optic tectum mRNA blots were hybridized with a chicken RGMa-specific probe (aa 869-1030), revealing major transcripts of 5.8 and $6.8 \mathrm{~kb}$. B, Mouse hippocampus mRNA blots were also hybridized with an RGMa-specific probe (aa 869-1030), revealing major transcripts of 5.8 and $6.8 \mathrm{~kb}$. Total RNA was obtained from E19, P5, P15, and adult $(A)$ hippocampus. A hybridization with a $2 \mathrm{~kb} \beta$-actin probe was used to confirm that identical mRNA levels are given in all lanes. Positions of RGMa mRNA are labeled with the respective transcript bands. $B$, At E18.5 the cortical plate is stained intensively. In the developing hippocampus the principal cell layers of CA1 and CA3 are labeled. C, In a control section from E18.5 incubated with an RGMa sense probe, no signal can be detected. D, In the hippocampus at $P 7$, strong mRNA expression is observed in the CA1 region, subiculum, and hilus. The dentate gyrus and the CA3 region show weak staining. $E$, This micrograph is a high-power magnification of the dentate gyrus shown in $D$. Note the intense staining of hilar mossy cells (arrows), the population of cells giving rise to the commissural-associational projection to the inner molecular layer. CA, Cornu ammonis; DG, dentate gyrus; gcl, granule cell layer; hi, hilus. Scale bars: $A, 500 \mu \mathrm{m} ; B, 400 \mu \mathrm{m} ; C, 600 \mu \mathrm{m} ; D, 300 \mu \mathrm{m}$; $E, 100 \mu \mathrm{m}$.

gradient along the anterior-posterior axis of the embryonic tectum (Monnier et al., 2002). In the same study the authors have demonstrated that recombinant RGMa induces collapse of temporal but not nasal growth cones of retinal ganglion cells and guides temporal retinal axons in vitro. These results point to a repulsive and axon-specific guiding activity of RGMa.

In the present study, we have examined the role of RGMa in the development of the entorhino-hippocampal system in rodents. In situ hybridization and immunohistochemistry show that RGMa is expressed during perinatal development in the hippocampus. Stripe and outgrowth assays reveal that entorhinal neurites are repelled by recombinant RGMa. Both separation of RGMa from its GPI anchor and specific blockade of the RGMa protein did not impair the outgrowth of entorhinal axons but resulted in an abnormal innervation of the hippocampal target. These results support a role for RGMa in the developmental construction of the precise entorhino-hippocampal connectivity pattern.

\section{Materials and Methods \\ Northern blotting}

Total RNA was prepared from frozen mouse hippocampus [embryonic day (E) 16.5, postnatal day (P) 5, P15, adult] by column purification (RNeasy, Qiagen, Hilden, Germany). RNA was size-separated on 1\% denaturing agarose gels ( $2.5 \mu \mathrm{g}$ per lane) and blotted onto nylon membranes (Hybond-N+, Amersham Biosciences, Braunschweig, Germany). Blots were hybridized with random-primed ([ $\left.\left.{ }^{32} \mathrm{P}\right] \mathrm{dCTP}\right)$ DNA probes encompassing the coding region of RGMa (accession number $\mathrm{BC} 023870$ ). Overnight hybridizations were performed at $60^{\circ} \mathrm{C}$ in the presence of $50 \%$ formamide, $5 \times$ Denhardt's solution, $5 \times$ SSC, $0.5 \%$ SDS, and $100 \mathrm{ng} / \mathrm{ml}$ probe. The final washing stringency was $0.1 \%$
SSC and $0.1 \%$ SDS at $60^{\circ} \mathrm{C}$. Blots were exposed to Amersham Hyperfilm ECL.

\section{In situ hybridization}

For in situ hybridization experiments, E16.5, E18.5, and P7 mouse brains were directly frozen in O.C.T. compound (Tissue-Tek). Sections of $20 \mu \mathrm{m}$ were prepared on a cryostat. Hybridization was performed with a digoxygenin (Dig)labeled antisense riboprobe corresponding to nucleotides 245-629 of the mouse RGMa cDNA. A sense probe served as control. In situ hybridization was performed as described (Oldekamp et al., 2004).

\section{Immunohistochemistry}

Postnatal mice (P7) were killed by transcardiac perfusion with $4 \%$ paraformaldehyde, $\mathrm{pH}$ 7.4. Brains were removed and postfixed overnight in the same fixative before vibratome sectioning. Immunohistological analysis was performed on coronal free-floating vibratome sections $(70 \mu \mathrm{m})$ incubated with a polyclonal rabbit antibody $(\mathrm{Ab})$ against the RGMa peptide EEVVNAVEDR spanning residues 279-289. This peptide appears in mouse as well as in chicken, and the resulting Ab has been demonstrated to only recognize RGM when used in Western blotting (Monnier et al., 2002).

All sections were incubated with $0.02 \%$ hydrogen peroxide for 10 min to block endogenous peroxidases and rinsed in PBS. Sections were then incubated with blocking solution [PBS containing $0.2 \%$ Triton X-100 and $10 \%$ normal goat serum (NGS)] for $30 \mathrm{~min}$. The RGMa Ab was diluted 1:500 in PBS, 0.1\% Triton $\mathrm{X}-100$, and $1 \% \mathrm{NGS}$, and sections were incubated at $4^{\circ} \mathrm{C}$ overnight. After washing with PBS, sections were incubated with a secondary Ab (biotinylated goat anti-rabbit IgG, diluted 1:250) with $1 \%$ NGS and $0.1 \%$ Triton X-100 for $2 \mathrm{hr}$ at room temperature. Sections were then washed and transferred to $\mathrm{ABC}$ solution (ABC-Elite, Vector Laboratories, Burlingame, $\mathrm{CA}$ ) for 2 hr. After final washes in PBS, sections were stained with $0.07 \% 3,3^{\prime}$ diaminobenzidine tetrahydrochloride (DAB) activated with $0.001 \%$ hydrogen peroxide in PBS, mounted onto gelatin-coated slides dehydrated through an ascending series of ethanol, and coverslipped. Sections were digitally photographed (Olympus BX-50).

\section{RGMa-alkaline phosphatase fusion protein binding}

RGMa-alkaline phosphatase (RGMa-AP) binding to receptor molecules was assayed using a ligand-affinity probe. Chicken RGMa-AP, which consisted of the extracellular domain of RGMa, was fused in-frame with a heat-stable human placental alkaline phosphatase (Genhunter). To detect expression of RGMa receptor proteins, mouse sections $(20 \mu \mathrm{m}$ thickness) from $\mathrm{P} 1$ were prepared on a cryostat and fixed with methanol at $-80^{\circ} \mathrm{C}$ for $5 \mathrm{~min}$. After the sections were rehydrated in PBS, they were equilibrated in HBSS without $\mathrm{Ca}^{2+} / \mathrm{Mg}^{2+}$ for 5 min and incubated in HBSS supplemented with $20 \%$ fetal calf serum (FCS) for $2 \mathrm{hr}$. The sections were then overlaid with concentrated conditioned medium containing the recombinant protein diluted in HBSS plus 20\% FCS for 90 min. After one wash with HBSS and three washes with TBS $(20 \mathrm{~mm}$ Tris- $\mathrm{HCl}, 135 \mathrm{~mm} \mathrm{NaCl}, \mathrm{pH}$ 7.5) for $5 \mathrm{~min}$ each, the sections were equilibrated with PBS for 5 min and fixed in $3.7 \%$ formaldehyde in PBS for $5 \mathrm{~min}$. After one wash with PBS, endogenous phosphatases were heatinactivated at $65^{\circ} \mathrm{C}$ for $50 \mathrm{~min}$. After equilibrating with AP-buffer (100 mm Tris, $100 \mathrm{~mm} \mathrm{NaCl,} 5 \mathrm{~mm} \mathrm{MgCl}_{2}$, pH 9.5), bound RGMa-AP fusion proteins were visualized with a staining solution containing 34 $\mathrm{mg} / \mathrm{ml} \mathrm{Nitro-blue-tetrazolium}$ and $18 \mathrm{mg} / \mathrm{ml}$ 5-bromo-4-chloro-3indolylphosphate (Boehringer Mannheim, Mannheim, Germany) in AP-buffer. The specificity of the RGMa-AP binding was determined by 
competition through an excess of unlabeled RGMa peptide, which abolished the binding.

\section{Explant preparation}

To collect embryonic tissue from E18.5, pregnant mice were anesthetized with Ketamin (5 $\mathrm{mg} / 100 \mathrm{mg}$ ), and embryos were removed from the uterus. Embryos were placed in cold HBSS medium (Invitrogen, Gaithersburg, MD) and decapitated, and hippocampi were dissected. Entorhinal explants were microdissected out with tungsten needles under binocular optics with $40 \times$ magnification. The explants were placed in suspension culture in a $5.5 \% \mathrm{CO}_{2}$ humidified incubator in Neurobasal medium (Invitrogen) supplemented with $100 \mathrm{U} / \mathrm{ml}$ penicillin, $100 \mu \mathrm{g} / \mathrm{ml}$ streptomycin, $10 \%$ heatinactivated fetal bovine serum, and B27 supplement (Invitrogen) for $2 \mathrm{hr}$ for equilibrium. Thereafter, entorhinal hippocampal explants were immediately used for the assays.

\section{Explant outgrowth assay}

Entorhinal cortex (EC) explants were plated on confluent layers of RGMa or mock-transfected cells. The total number of $\beta$-III tubulin-stained (TUJ-1 Ab, Convance) processes from the entorhinal explants was scored semiquantitatively.

\section{Stripe assay}

Preparation of membranes. Membrane suspensions were prepared from stably RGMatransfected human embryonic kidney (HEK) 293 cells. All solutions used were sterile, $4^{\circ} \mathrm{C}, \mathrm{pH} 7.4$, and supplemented with protease inhibitors (protease inhibitor mixture; Sigma, St. Louis, MO). Cells were homogenized in homogenization buffer ( $10 \mathrm{~mm}$ Tris- $\mathrm{HCl}, \mathrm{pH} 7.4,1.5 \mathrm{~mm} \mathrm{CaCl}_{2}$, and $1 \mathrm{~mm}$ spermidine) by pressing the tissue at least 10 times through 27 ga, 1.5 syringe needles. The homogenate was layered on top of a step gradient of 50 and $5 \%$ sucrose and centrifuged for $10 \mathrm{~min}$ at $28,000 \times g$ at $4^{\circ} \mathrm{C}$ in a TLS 55 rotor (Beckman Instruments, Fullerton, CA). The membrane fragments were collected from the interphase layer situated between 5 and $50 \%$ sucrose and washed with PBS. Membrane suspensions were adjusted to an optical density of 0.1 (measured at $220 \mathrm{~nm})$.

Preparation of stripes. Stripe preparation was performed according to the protocol described previously (Walter et al., 1987). Stripes were prepared with membranes obtained from either RGMa or mock-transfected HEK293 cells onto polyvinyl propylene filters with $0.4 \mu \mathrm{m}$ pores precoated with $20 \mu \mathrm{g} / \mathrm{ml}$ laminin. The membrane carpets were then placed into cell culture inserts (Falcon) for $35 \mathrm{~mm}$ tissue culture dishes with 1.5 $\mathrm{ml}$ culture medium. Explants were positioned on the membrane carpets using sterile forceps. Cultures were maintained in a $5.5 \% \mathrm{CO}_{2}$ humidified incubator for $5 \mathrm{~d}$.

\section{Analysis of outgrowth preference}

Neurites growing out from the explants were visualized by immunolabeling with a neurofilament $\mathrm{Ab}$ (Boehringer Mannheim) and subsequent $\mathrm{DAB}$ staining as described above. Culture medium was removed from the dishes $5 \mathrm{~d}$ after the explants were placed on the carpets. Neurite growth from the explants was examined and photographed by light microscope. Growth preferences for one or the other set of membrane stripes were evaluated using a three-class system: (1) clear-cut preference, with almost all of the fibers growing on one of the membrane lanes, (2) slight or moderate preference, with most fibers growing preferentially on one membrane lane, although others cross randomly, and (3) no choice or random outgrowth.

\section{Preparation of organotypic slice cultures}

Entorhinal cortex with the adjacent hippocampus was dissected from newborn mice (P0) and cut into $350 \mu \mathrm{m}$ sections with a tissue chopper.
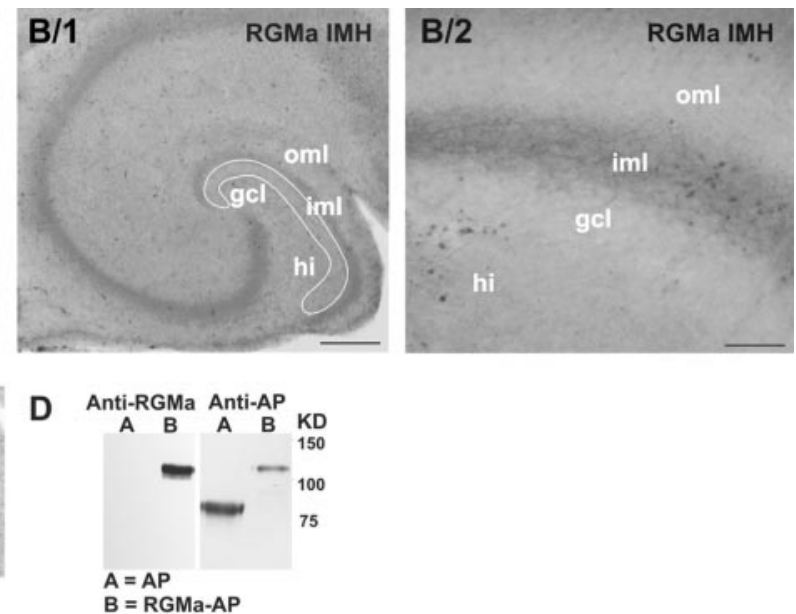

$B=R G M-A P$

Figure 2. Entorhinal cortex tracing, RGMa immunohistochemistry, and binding. $A$, The biocytin-labeled entorhinal projection shows its typical distribution in the dentate gyrus. Labeled axons are found exclusively in the outer molecular layer, whereas the

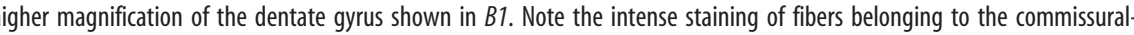
gelecular layer. Binding was also seen in the stratum radiatum-stratum lucidum. hi, Hilus; EC, entorhinal cortex; gcl, granular cell layer; oml, outer molecular layer; iml, inner molecular layer. D, An RGMa-specific Ab (anti$\mathrm{RGMa}$ ) recognizes the $113 \mathrm{kDa}$ RGMa-AP fusion protein, whereas an alkaline phosphatase (anti-AP) Ab detects an $80 \mathrm{kDa}$ protein. .

These complex slices $(n=62)$ were cultivated on porous membranes (Millicell, Millipore Corporation, Bedford, MA) for $7 \mathrm{~d}$ in vitro (DIV 7) and fed with medium as described previously (Ceranik et al., 1999). The cocultures were treated in different sets of experiments either with a functional RGMa Ab $(1 \mu \mathrm{g} / \mathrm{ml})(n=18)$ or with phosphatidylinositolspecific phospholipase C (PI-PLC) (Sigma) at two different concentrations $(5$ and $10 \mathrm{U} / \mathrm{ml})(n=36)$. Treatment of cultures was performed during the entire incubation period. During this time, medium and drugs were replaced every second day. No substances were added to control cultures $(n=8)$. At DIV 7 the developed entorhino-hippocampal projection of all cultured slices was traced using biocytin. After reincubation for 24-36 hr to allow for anterograde transport of the tracer, the cocultures were fixed with $4 \%$ paraformaldehyde, $0.1 \%$ glutaraldehyde, and $15 \%$ saturated picric acid in $0.1 \mathrm{M}$ phosphate buffer $(\mathrm{PB})$ at $\mathrm{pH} 7.4$ for $2 \mathrm{hr}$. Thereafter, all cocultures were resliced on a vibratome $(50 \mu \mathrm{m})$, permeabilized with $0.1 \%$ Triton X-100 for $30 \mathrm{~min}$ in $\mathrm{PB}$, and incubated with ABC-Elite complex (1:50; Vector Laboratories) overnight at $4^{\circ} \mathrm{C}$. Sections were developed using a nickel-cobalt-intensified DAB protocol (Schwab et al., 2000). Labeled sections were counterstained with cresyl violet, dehydrated, coverslipped with Entellan, and digitally photographed. A blinded descriptive semiquantitative analysis of the entorhino-hippocampal projection was performed by three independent investigators using the following criteria: ++ , layer-specific termination, +- , layer-specific termination with some aberrant fibers, - , complete loss of layer specificity.

For blocking experiments, a chicken RGMa peptide spanning residues 195-349 was used to generate rabbit antibodies. For Ab purification, sera were applied to a protein-G-agarose column (Amersham Biosciences). After extensive washing with PBS, bound IgG was eluted with $0.1 \mathrm{M}$ glycine, $0.15 \mathrm{M}$ $\mathrm{NaCl}, \mathrm{pH} 2.5$, immediately neutralized, and dialyzed against PBS.

\section{Results}

RGMa is expressed predominantly in the hippocampus during perinatal stages of development

To determine whether RGMa is expressed during development of the CNS, we examined the sites of expression and applied Northern blotting, in situ hybridization, and immunohistochemistry.

Northern blot analysis of RGMa in the mouse CNS detected 

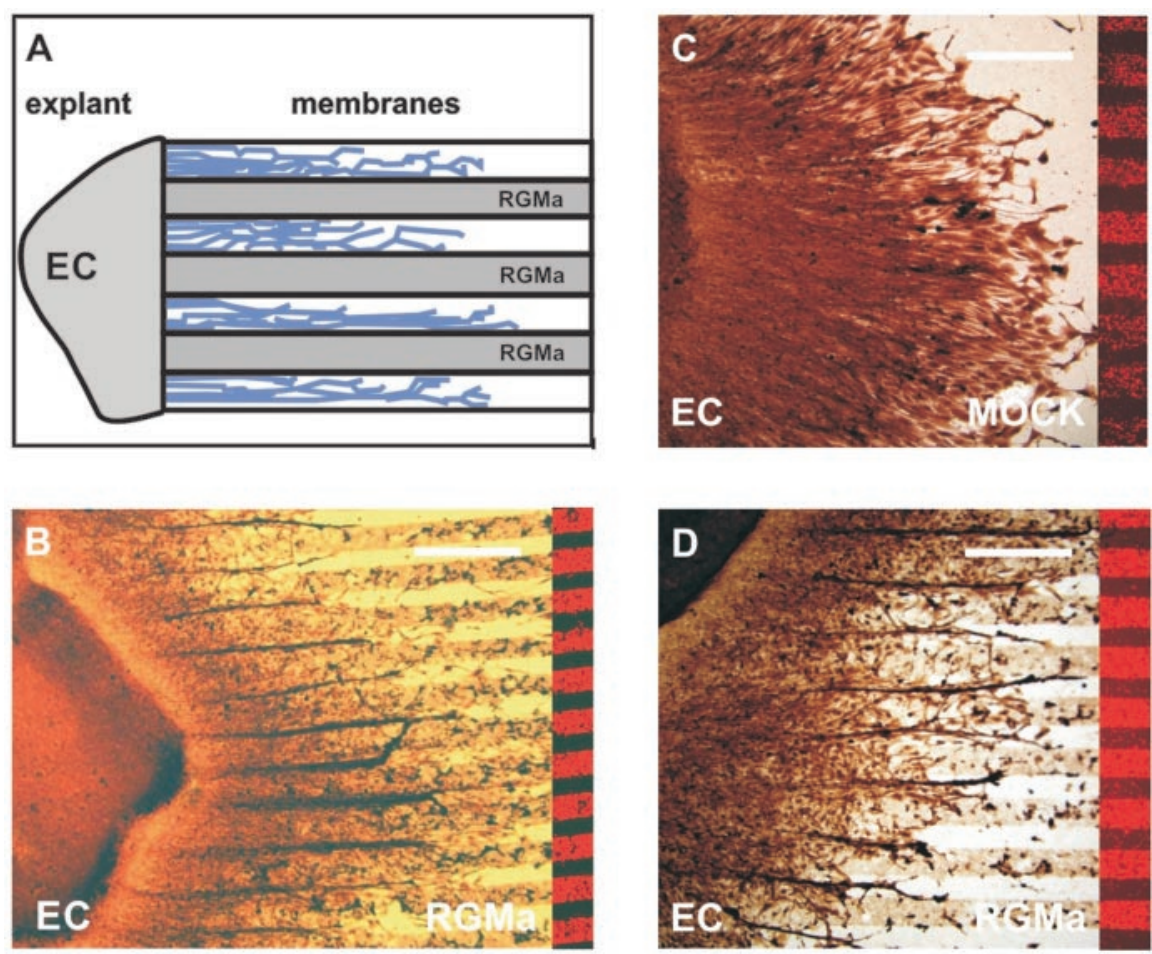

\begin{tabular}{|l|l|c|l|l|}
\hline \begin{tabular}{l} 
Category of ax on choice \\
\hline $\begin{array}{l}\text { substrates } \\
\text { compared }\end{array}$
\end{tabular} $\begin{array}{l}\text { ec-explants } \\
\text { n }\end{array}$ & no preference & $\begin{array}{l}\text { slight } \\
\text { preference }\end{array}$ & $\begin{array}{l}\text { clear-cut } \\
\text { preference }\end{array}$ \\
\hline RGMa/MOCK & 30 & 0 & 13 & 17 \\
\hline MOCK/MOCK & 30 & 22 & 8 & 0 \\
\hline
\end{tabular}

Figure 3. The growth preference of entorhinal neurites is influenced by RGMa. $A$, Schematic illustration of neurites avoiding RGMa lanes on a membrane carpet growing out of an entorhinal cortex explant. B, D, Entorhinal neurites of E19 explants avoid RGMa membrane lanes but do not display any preference when offered a choice of membrane lanes from mock-transfected cells (C).E, Table illustrating categories of axon choice from entorhinal neurites on RGMa and control substrates. Scale bars: $B, 250 \mu \mathrm{m}$; $C, D, 100 \mu \mathrm{m}$.

two bands of 5.8 and $6.8 \mathrm{~kb}$ with molecular weight similar to the bands detected in the chick tectum (Monnier et al., 2002) (Fig. $1 A)$. In the hippocampus, strong hybridization signals were obtained at E18.5 and P5. During adulthood, the signal decreased to background levels.

To obtain further insight into the possible function of RGMa in the developing entorhino-hippocampal formation, the spatiotemporal distribution of the RGMa mRNA was investigated using a nonradioactive in situ hybridization. At E18.5, RGMa mRNA was strongly expressed in the parietal cortical plate, in the $\mathrm{CA} 1$ and CA3 regions and in the hilus of the hippocampus (Fig. $1 B$ ).

At P7, a strong Dig-labeling was observed, particularly in many large hilar neurons, presumably mossy cells (Fig. 1D,E). These cells give rise to the commissural projection to the inner molecular layer of the dentate gyrus. The RGMa signal was also found in the CA1 region, whereas other hippocampal cell layers were only faintly stained.

\section{Localization of the RGMa protein in the hippocampus}

On the basis of mRNA expression patterns in the developing hippocampal formation, the localization of the RGMa protein was studied in detail. Immunostaining of the hippocampus derived from postnatal brain (P7) was most prominent in the inner molecular layer of the dentate gyrus (Fig. 2 B1,B2). In this layer, axonal profiles appeared to be labeled, indicating an axonal localization of RGMa on commissural-associational fibers. The granule cell layer and the outer molecular layer, which is occupied by perforant path fibers, were devoid of any immunostaining.

\section{Localization of the RGMa ligand protein} in the hippocampus

Because RGMa was expressed in the inner molecular layer of the hippocampus, we went further on and studied the distribution of the RGMa receptor protein. To detect RGMa receptor binding in the hippocampus, we stained P1 mouse brain sections with a ligand-affinity probe, which consisted of the extracellular domain of RGMa fused in frame with a human placental AP (Fig. 2C). Binding in the hippocampus at $\mathrm{P} 1$ was most prominent in the dendritic fields of the CA3 and dentate gyrus. No staining was observed on principal neurons of the dentate gyrus and the hippocampus proper. The strongest RGMa-AP binding signal was observed in the marginal zone of the dentate gyrus, suggesting the presence of the corresponding receptor on entorhinal axons that have already entered the developing dentate gyrus. Binding also occurred in the stratum lucidum of CA3, the prospective termination zone of the mossy fiber tract, and in the stratum radiatum of $\mathrm{CA} 3$ and $\mathrm{CA} 1$ (Fig. 2C).

\section{Growth preference of EC neurites is controlled by RGMa}

We next examined whether recombinant RGMa is able to guide entorhinal axons in vitro. To address this question, the well characterized stripe assay was used. Growth cones were exposed to two kinds of stripes containing particles from RGMa- or mocktransfected cells. Entorhinal cortex explants obtained from E18.5 were allowed to grow on these carpets of alternating membrane stripes (Fig. 3). Outgrowing entorhinal neurites tended to fasciculate and clearly avoided membranes prepared from RGMaexpressing cells (Fig. 3 B, D). In contrast, given the choice between a membrane lane from mock-transfected cells or laminin, embryonic entorhinal neurites did not display any preference and crossed lane borders freely (Fig. 3C). In all experimental groups, many migrating cells could also be observed, but these did not influence axonal outgrowth. These results support the finding that extending neurites from the entorhinal cortex are selectively repelled by RGMa. This effect was estimated semiquantitatively (Fig. 3E).

\section{Explant outgrowth assay}

Entorhinal explants from E18.5 mouse brain were cultivated on a confluent monolayer of HEK293 cells expressing RGMa $(n=25)$ or transfected with the expression vector alone $(n=25)$ (Figs. 4 , 
$5)$. The expression of the ligand was further confirmed by staining membrane extracts obtained from the RGMatransfected cells with an RGMa-specific $\mathrm{Ab}$, detecting a single band of the expected size (data not shown). In particular, the outgrowth of entorhinal neurites was strongly inhibited by RGMa (Fig. 4D-F) compared with the length of entorhinal explant outgrowth on mock-transfected HEK293 cells (Fig. 4A-C). In contrast, RGMa did not inhibit the neurite outgrowth from control explants $(n=15)$ (Fig. $4 M-O$ ). The treatment of RGMatransfected HEK293 cells with an RGMaspecific $\mathrm{Ab}(3 \mu \mathrm{g} / \mathrm{ml})$ during entorhinal explant culture $(n=25)$ completely abolished the outgrowth inhibitory effect of RGMa (Fig. 4G-I). Because RGMa can be removed from the membrane by PI-PLC treatment $(1 \mathrm{U} / \mathrm{ml})$, we analyzed the effect of PI-PLC on RGMa-transfected HEK293 cell inhibition of entorhinal neurite outgrowth $(n=25)$. Removal of RGMa from the cell membranes restored neurite elongation from entorhinal explants (Fig. $4 J-L$ ). This observation suggests that RGMa selectively and specifically inhibits entorhinal axon outgrowth. The neurite outgrowth was analyzed semiquantitatively (Fig. 5).

\section{Blockade of RGMa leads to loss of target} recognition of entorhinal axons

To address the function of RGMa in a more in vivo-like environment, organotypic entorhino-hippocampal complex cocultures taken at P0 were incubated for $8 \mathrm{~d}$ with either the enzyme PI-PLC, which cleaves the GPI-anchor, or a blocking Ab against RGMa. By use of the anterograde tracer biocytin, the entorhinal projections that had developed under control conditions and after drug treatment could be monitored in all cocultured slices (Fig. 6). Disruption of the RGMa function did not prevent entorhinal axons to find their correct route to the hippocampal target culture; however, it led to failure of entorhinal axons to recognize their appropriate target zone in the dentate gyrus. Axons were no longer confined to the outer molecular layer and invaded the inner molecular layer (Fig. 6D,E). These changes were similar at all tested concentrations of PIPLC, as illustrated in the diagram in Figure $6 F$. Furthermore, when incubated with the neutralizing $\mathrm{Ab}$, the entorhinal projection was massively altered, and numerous biocytin traced fibers not only invaded the inner molecular layer but also passed the granule cell layer and spread throughout the hilar region of the hippocampus. (Fig. 6E). More than $50 \%$ of all RGMa Ab-treated slices developed a completely perturbed entorhinal projection (Fig. 6G). In the control cocultures, Sytox-Green.

\section{3-monolayer/ EC-explant}
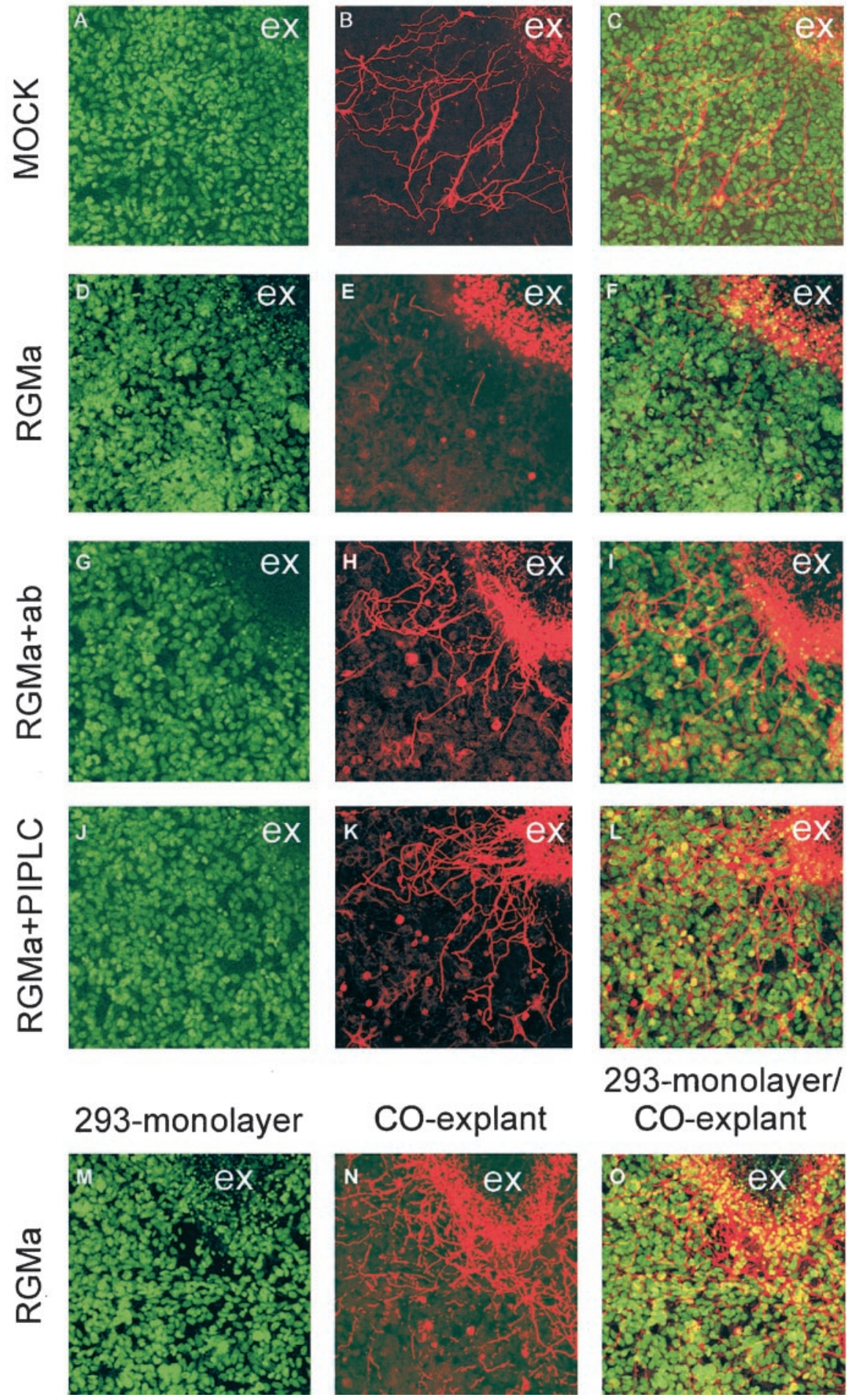

Figure 4. Outgrowth of entorhinal neurites is inhibited by RGMa. E18.5 entorhinal cortex explants were supercultivated on a confluent layer of 293 mock-transfected $(A-C)$, RGMa-transfected $(D-F)$, or RGMa-transfected cells treated with an RGM Ab $(G-l)$ or PI-PLC $(1 \mathrm{U} / \mathrm{ml})(J-L)$. Neurites were stained with $\beta$-III tubulin Ab (red), and cells were counterstained with

biocytin-traced entorhinal projection showed an intense labeling in the correct termination zone, the outer two-thirds of the molecular layer of the dentate gyrus (Fig. 6C). A few biocytin-labeled fibers were seen in the inner molecular layer. 
A

\begin{tabular}{|c|c|c|c|c|}
\hline & & \multicolumn{3}{|c|}{} \\
\hline 293-cells & EC-explants & \multicolumn{3}{|c|}{$\mathbf{n}$ of outgrown neurites } \\
\hline & $\mathbf{n}$ & $\mathbf{+}$ & $\mathbf{+ +}$ & $\mathbf{+ + +}$ \\
\hline RGMa & $\mathbf{2 5}$ & 36 & 15 & 3 \\
\hline MOCK & $\mathbf{2 5}$ & 178 & 179 & 167 \\
\hline RGMa + ab & $\mathbf{2 5}$ & 139 & 196 & 72 \\
\hline PIPLC & $\mathbf{2 5}$ & 109 & 169 & 85 \\
\hline CO-explant & $\mathbf{1 5}$ & 186 & 256 & 167 \\
\hline
\end{tabular}

B

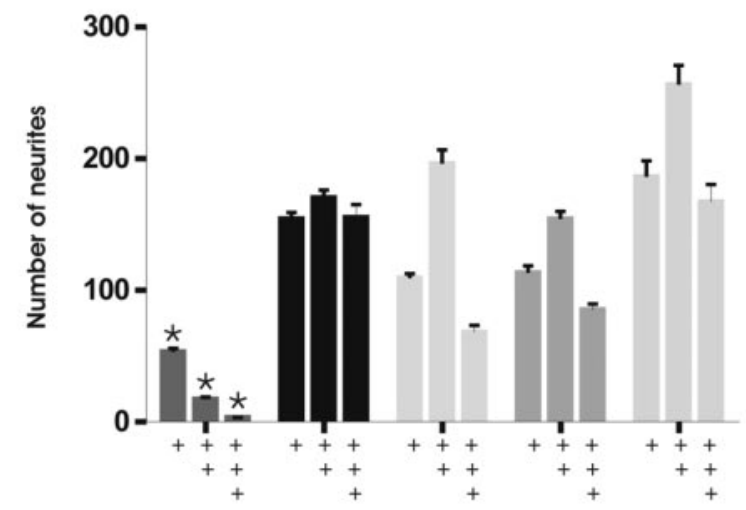

Figure 5. Quantification of entorhinal neurite outgrowth as presented in Figure $4.0=$ no outgrowth; $+=$ low outgrowth $(\sim 100 \mu \mathrm{m}) ;++=$ moderate outgrowth $(\sim 200 \mu \mathrm{m}) ;+++=$ strong outgrowth $(\sim 500 \mu \mathrm{m})$; error bars indicate SEM; RGMa compared with all other groups $p<0.005$ (ANOVA; Mann-Whitney $U$ test).

\section{Discussion}

The formation of afferent connections in the dentate gyrus is controlled by an assembly of different guidance molecules. In this study we were able to identify RGMa, a novel GPI-linked axon guidance molecule in the developing entorhino-hippocampal system. In vitro RGMa exerts a repellent action on outgrowing entorhinal axons. RGMa mRNA expression was found in the cornu ammonis of the hippocampus and in the hilar region, the origin of the commissural-associational projection to the inner molecular layer of the dentate gyrus. Immunohistochemistry for RGMa showed that the protein is present in the inner molecular layer, presumably on commissural-associational hilar mossy cell axons. In contrast, strong RGMa binding was present in the outer molecular layer, the termination zone of the entorhinal projection. Disruption of the RGMa function led to a perturbed laminar innervation of the entorhinal projection in organotypic slice culture experiments. This is of great interest because the precise laminar segregation of extrinsic and intrinsic afferents, a common feature of the synaptic architecture in the hippocampus, is an accepted model for studying the molecular mechanisms underlying the wiring of the cortex.

In the hippocampus, there is a sequential ingrowth of afferents into the dentate gyrus as entorhinal axons precede and RGMapositive commissural-associational axons follow (Frotscher and Heimrich, 1993; Super and Soriano, 1994). The functional stripe and outgrowth assays demonstrate a repulsive effect of RGMa on entorhinal axons. Thus, first, entorhinal axons that arrive at E19 in the RGMa-free dentate gyrus are not repelled but are enabled to occupy their target area later in development, at $\mathrm{P} 2$, when commissural-associational axons invade the inner molecular layer of the dentate gyrus. This is accompanied by the expansion of RGMa receptor-loaded entorhinal fibers toward the inner molecular layer, which might be prevented by RGMa expressed on the commissural-associational axons. This subsequent appear-

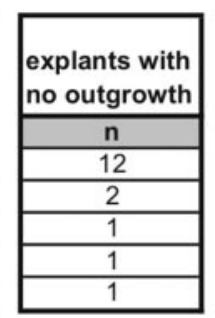

ance of the RGMa-containing axons suggests that in the developing dentate gyrus, RGMa is involved in both the maintenance and stabilization of the earlier-formed laminated entorhinal projection via its repulsive action on these fibers. This hypothesis is supported by the fact that blocking the RGMa protein or cleavage of the GPI anchor resulted in a remarkable loss of the layer-specific termination of entorhinal afferents in the dentate gyrus. This is demonstrated by an aberrant ingrowth of entorhinal fibers into the inner molecular layer of the dentate gyrus after PI-PLC treatment and even further into other hippocampal subfields as observed after incubation of the cocultures with the neutralizing RGMa Ab.

Our results together with previous studies by Pozas et al. (2002) and Gu et al. (2003) indicate that a network of guiding and target-related molecules are involved in the laminar segregation of EC projections to the hippocampus. Moreover, these studies suggest that EC neurons respond differentially to guidance cues: although RGMa seems to be involved in the laminar segregation pattern of entorhinal fibers terminating in the outer molecular layer of the dentate gyrus, semaphorin (Sema) 3a seems to be involved mainly in the guidance of entorhinal projections toward the stratum lacunosum moleculare of the CA1 region, as shown in Sema 3a -/- mice (Pozas et al., 2002).

It is well known that the laminar termination of afferents in the marginal zones of the hippocampus and the dentate gyrus is a suitable model for the analysis of pathfinding, target recognition, and sprouting processes after denervation (Li et al., 1993, 1994; Woodhams and Atkinson, 1996; Del Rio et al., 1997; Frotscher, 1997). The molecular signals necessary for the formation of the normotypic entorhino-dentate projection are present in hippocampal tissue maintained in organotypic coculture with explants of the EC (Li et al., 1993, 1994; Woodhams and Atkinson, 1996; Frotscher, 1997). These experiments show that laminaspecific molecules in the hippocampal fields play important roles in the formation of layer-specific afferent projections; however, none of these studies provided information on the mechanisms guiding entorhinal axons along their normal pathways and into their target fields.

Ablation of reelin-secreting hippocampal Cajal-Retzius cells (CR) prevents entorhinal fiber ingrowth into the hippocampus in vitro (Borrell et al., 1999). In the hippocampus of reeler mice lacking reelin, entorhinal fibers terminate with correct laminar specificity, indicating that the positioning of CR cells plays a key role in guiding entorhinal axons to their target layer (Förster et al., 1998; Borrell et al., 1999; Deller et al., 1999). Furthermore, experiments with membrane preparations indicate the presence of adhesive, attractive, and repulsive properties in entorhinohippocampal tissue underlying the formation of the layered termination (Skutella et al., 1999; Förster et al., 2001). On a molecular level, the laminated distribution of hippocampal afferents is probably the result of a cooperation between attractive and repulsive guidance factors. Previously, this function was attributed to semaphorins-plexins-neuropilins (Chen et al., 2000; Cheng et 
al., 2001; Pozas et al., 2002; Gu et al., 2003), the slits-robo system (Nguyen Ba-Charvet et al., 1999), netrins (Steup et al., 2000; Heimrich et al., 2002), ephrin ligands, Eph receptor tyrosine kinases (Stein et al., 1999), and ECM molecules (Förster et al., 2001; Zhao et al., 2003).

The expression pattern of RGMa in the hippocampal formation provides the first evidence that this molecule might be involved in the targeting and wiring of intrahippocampal projections; however, it will require further investigation to determine in more detail whether subpopulations of hippocampal neurons respond differentially to this guidance cue.

In summary, our observations indicate that RGMa, first described in the tectal system of chicken, has a similar function as a repulsive signal in the rodent cortex. A close characterization of RGMa therefore improves our understanding of the formation of hippocampal connections, especially the stabilization of the laminar organization of afferent fiber systems in the dentate gyrus. This will increase our knowledge of the molecular mechanisms of axon guidance and target recognition of cortical neurons.

\section{References}

Bayer SA, Altman J (1987) Directions in neurogenetic gradients and patterns of anatomical connections in the telencephalon. Prog Neurobiol 29:57-106.

Borrell V, Del Rio JA, Alcantara S, Derer M, Martinez A, D’Arcangelo G, Nakajima K, Mikoshiba K, Derer P, Curran T, Soriano E (1999) Reelin regulates the development and synaptogenesis of the layer-specific entorhino-hippocampal connections. J Neurosci 19:1345-1358.

Ceranik K, Deng J, Heimrich B, Lubke J, Zhao S, Forster E, Frotscher M (1999) Hippocampal Cajal-Retzius cells project to the entorhinal cortex: retrograde tracing and intracellular labelling studies. Eur J Neurosci 11:4278-4290.

Chen H, Bagri A, Zupicich JA, Zou Y, Stoeckli E, Pleasure SJ, Lowenstein DH, Skarnes WC, Chedotal A, Tessier-Lavigne M (2000) Neuropilin-2 regulates the development of selective cranial and sensory nerves and hippocampal mossy fiber projections. Neuron 25:43-56.

Cheng HJ, Bagri A, Yaron A, Stein E, Pleasure SJ, Tessier-Lavigne M (2001) Plexin-A3 mediates semaphorin signaling and regulates the development of hippocampal axonal projections. Neuron 32:249-263.

Deller T, Drakew A, Heimrich B, Förster E, Tielsch A, Frotscher M (1999) The hippocampus of the reeler mutant mouse: fiber segregation in area CA1 depends on the position of the postsynaptic target cells. Exp Neurol 156:254-267.

Del Rio JA, Heimrich B, Borrell V, Förster E, Drakew A, Alcantara S, Nakajima K, Miyata T, Ogawa M, Mikoshiba K, Derer P, Frotscher M, Soriano E (1997) A role for Cajal-Retzius cells and reelin in the development of hippocampal connections. Nature 385:70-74.

Förster E, Kaltschmidt C, Deng J, Cremer H, Deller T, Frotscher M (1998) Lamina-specific cell adhesion on living slices of hippocampus. Development 125:3399-3410.
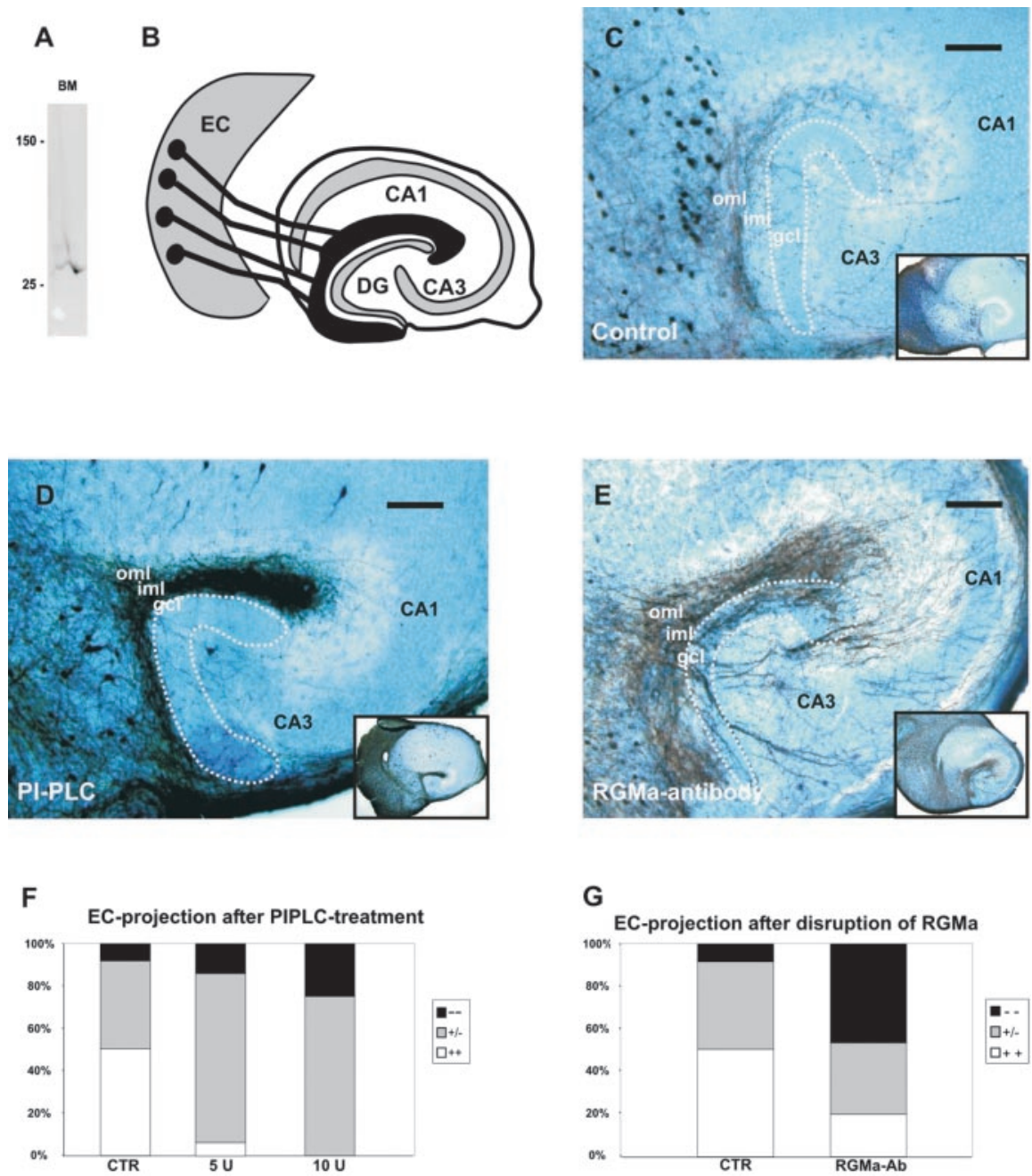

Figure 6. RGMa controls targeting of entorhinal fibers in organotypic slice culture. $A$, To ensure Ab specificity, Western blotting was performed on membrane extracts. The Ab recognizes a $33 \mathrm{kDa}$ band, which is the size of RGMa. $B$, Schema of the entorhinoexplant. C, Biocytin-traced control coculture at DIV 9. Labeled entorhinal axons had developed their normal termination pattern and are almost confined to the outer molecular layer (oml) of the dentate gyrus. The termination zone of the commissuralreveals that an entorhino- hippocampal projection has developed. Note that labeled entorhinal axons are no longer confined to their normal termination zone. Entorhinal axons have invaded the entire marginal zone (iml + oml) of the dentate gyrus. $E$, An

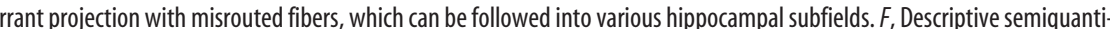
with a specific Ab against RGMa. Approximately $50 \%$ (black bar) of all treated cultures show a massive aberrant entorhina projection. CA1, CA3 hippocampal subfields; gcl, granule cell layer; + + , layer specific termination; + - , layer specific termination with some aberrant fibers; - - complete loss of layer specificity. Scale bars: $C-E, 100 \mu \mathrm{m}$.

Förster E, Zhao S, Frotscher M (2001) Hyaluronan-associated adhesive cues control fiber segregation in the hippocampus. Development 128:3029-3039.

Frotscher M (1997) Dual role of Cajal-Retzius cells and reelin in cortical development. Cell Tissue Res 290:315-322.

Frotscher M, Heimrich B (1993) Formation of layer-specific fiber projections to the hippocampus in vitro. Proc Natl Acad Sci USA 90:10400-10403.

Gu C, Rodriguez ER, Reimert DV, Shu T, Fritzsch B, Richards LJ, Kolodkin AL, Ginty DD (2003) Neuropilin-1 conveys Semaphorin and VEGF signaling during neural and cardiovascular development. Dev Cell 5:45-57.

Heimrich B, Vogt J, Simbürger E, Skutella T, Glumm R (2002) Axon guidance and the formation of specific connections in the hippocampus. Neuroembryology 1:154-160.

Li D, Field PM, Starega U, Li Y, Raisman G (1993) Entorhinal axons project 
to dentate gyrus in organotypic slice co-culture. Neuroscience 52:799-813.

Li D, Field PM, Yoshioka N, Raisman G (1994) Axons regenerate with correct specificity in horizontal slice culture of the postnatal rat entorhinohippocampal system. Eur J Neurosci 6:1026-1037.

Monnier PP, Sierra A, Macchi P, Deitinghoff L, Andersen JS, Mann M, Flad M, Hornberger MR, Stahl B, Bonhoeffer F, Mueller BK (2002) RGM is a repulsive guidance molecule for retinal axons. Nature 419:392-395.

Nguyen Ba-Charvet KT, Brose K, Marillat V, Kidd T, Goodman CS, TessierLavigne M, Sotelo C, Chedotal A (1999) Slit2-mediated chemorepulsion and collapse of developing forebrain axons. Neuron 22:463-473.

Oldekamp J, Krämer N, Alvarez-Bolado G, Skutella T (2004) Expression pattern of the repulsive guidance molecules RGM A, B and C during mouse development. Gene Expr Patterns, in press.

Pozas E, Pascual M, Nguyen Ba-Charvet KT, Guijarro P, Sotelo C, Chedotal A, Del Rio JA, Soriano E (2002) Age-dependent effects of secreted semaphorins $3 \mathrm{~A}, 3 \mathrm{~F}$, and $3 \mathrm{E}$ on developing hippocampal axons: in vitro and phenotype of semaphoring $3 \mathrm{~A}(-/-)$ mice. Mol Cell Neurosci 18:26-43.

Schmidtmer J, Engelkamp D (2004) Isolation and expression pattern of three mouse homologous of chick RGM. Gene Expr Patterns 4:105-110.

Schwab MH, Bartholomae A, Heimrich B, Feldmeyer D, Druffel-Augustin S, Goebbels S, Nava FJ, Zhao S, Frotscher M, Tsai MJ, Nave KA (2000) Neuronal basic helix-loop-helix proteins (NEX and BETA2/Neuro D) regulate terminal granule cell differentiation in the hippocampus. J Neurosci [Erratum (2000) 20:8227] 20:3714-3724.
Skutella T, Nitsch R (2001) New molecules in hippocampal development and reorganization. Trends Neurosci 24:107-113.

Skutella T, Savaskan NE, Ninnemann O, Nitsch R (1999) Pathfinding of entorhinal fibers is dependent on target- and maturation specific membrane-bound molecules. Dev Biol 211:277-292.

Stahl B, Müller B, von Boxberg Y, Bonhoeffer F (1990) Biochemical characterization of a putative axonal guidance molecule of the chick visual system. Neuron 5:735-743.

Stein E, Savaskan NE, Ninnemann O, Nitsch R, Zhou R, Skutella T (1999) A role for the Eph ligand ephrin-A3 in entorhino-hippocampal targeting. J Neurosci 19:8885-8893.

Steup A, Lohrum M, Hamsho N, Savaskan NE, Ninnemann O, Nitsch R, Fujisawa H, Püschel AW, Skutella T (2000) Semaphorin3C and netrin-1 differentially affect axon growth in the hippocampal formation. Mol Cell Neurosci 15:141-155.

Super H, Soriano E (1994) The organization of the embryonic and early postnatal development murine hippocampus. II. Development of entorhinal, commissural and septal connections studied with the lipophilic tracer DiI. J Comp Neurol 344:101-120.

Walter J, Kern Veits B, Huf J, Stolze B, Bonhoeffer F (1987) Recognition of position-specific properties of tectal cell membranes by retinal axons in vitro. Development 101:685-696.

Woodhams PL, Atkinson DJ (1996) Entorhinal axons perforate hippocampal field CA3 in organotypic slice culture. Brain Res Dev Brain Res 95:144.

Zhao S, Forster E, Chai X, Frotscher M (2003) Different signals control laminar specificity of commissural and entorhinal fibers to the dentate gyrus. J Neurosci 23:7351-7357. 